\title{
Sperm Storage Costs Determine Survival and Immunocompetence in Newly Mated Queens of the Leaf-Cutting Ant Atta colombica
}

\author{
Barbara Baer-Imhoof ${ }^{1 *}$, Susanne P. A. den Boer ${ }^{2,3}$, Jacobus J. Boomsma ${ }^{3}$ and \\ Boris Baer ${ }^{1}$ \\ 1 Department of Entomology, Center for Integrative Bee Research, University of California, Riverside, Riverside, CA, \\ United States, ${ }^{2}$ Information Security Unit, Research and Innovation, University of Copenhagen, Copenhagen, Denmark, \\ ${ }^{3}$ Department of Biology, Centre for Social Evolution, University of Copenhagen, Copenhagen, Denmark
}

\section{OPEN ACCESS}

Edited by:

Alison McAfee

North Carolina State University,

United States

Reviewed by:

Ben Hopkins,

University of California, Davis,

United States

Serge Aron,

Université Libre de Bruxelles, Belgium

*Correspondence:

Barbara Baer-Imhoof barbarai@ucr.edu

Specialty section:

This article was submitted to

Ecophysiology,

a section of the journal

Frontiers in Ecology and Evolution

Received: 16 August 2021 Accepted: 25 November 2021

Published: 04 January 2022

Citation:

Baer-Imhoof B, den Boer SPA, Boomsma JJ and Baer B (2022) Sperm Storage Costs Determine Survival and Immunocompetence

in Newly Mated Queens of the Leaf-Cutting Ant Atta colombica.

Front. Ecol. Evol. 9:759183. doi: 10.3389/fevo.2021.759183
In the leaf-cutting ant Atta colombica, queens receive ejaculates from multiple males during one single mating event early in their lives. A queen's fertility and fitness therefore depend on maximizing the number of sperm cells she can store and maintain inside her spermatheca. Previous studies implied significant physiological mating costs, either originating from energetic investments maximizing sperm survival, or from resolving sexual conflicts to terminate male-driven incapacitation of rival sperm via serine proteases found in seminal fluid. Here we conducted an artificial insemination experiment, which allowed us to distinguish between the effects of sperm and seminal fluid within the queen's sexual tract on her survival and immunocompetence. We found significantly higher mortality in queens that we had inseminated with sperm, independently of whether seminal fluid was present or not. Additionally, after receiving sperm, heavier queens had a higher probability of survival compared to lightweight queens, and immunocompetence decreased disproportionally for queens that had lost weight during the experiment. These findings indicate that queens pay significant physiological costs for maintaining and storing sperm shortly after mating. On the other hand, the presence of seminal fluid within the queens' sexual tract neither affected their survival nor their immunocompetence. This suggests that the energetic costs that queens incur shortly after mating are primarily due to investments in sperm maintenance and not costs of terminating conflicts between competing ejaculates. This outcome is consistent with the idea that sexually selected traits in social insects with permanent castes can evolve only when they do not affect survival or life-time fitness of queens in any significant way.

Keywords: mating costs, leaf-cutting ant, Atta colombica, sperm competition, female choice, polyandry, costs of reproduction

\section{INTRODUCTION}

During mating, males transfer ejaculates consisting of both sperm and seminal fluid to females (Birkhead and Moller, 1998). The latter is a complex mixture of proteins and metabolites produced by several glands in the male's reproductive tract (Kubli, 2003; Poiani, 2006; Avila et al., 2011). These molecules maximize sperm viability and have anti-microbial properties. In polyandrous species, 
where females mate with multiple males in short succession, ejaculates from several potential sires overlap in time and space inside a female's sexual tract. As a consequence, conflicts over paternity can result in sperm competition (Parker, 1970), where rival ejaculates compete for sperm storage and/or direct egg fertilization (Simmons and Siva-Jothy, 1998; Parker and Pizzari, 2010). Females can also influence paternity by discriminating against sperm of less preferred males via cryptic female choice (Eberhard, 1996). In red junglefowl Gallus gallus for example, females eject sperm of subdominant males (Pizzari and Birkhead, 2000) and bias fertilizations toward sperm of unrelated males (Løvlie et al., 2013). However, reproductive traits under sexual selection are expected to incur physiological costs, which trade off with other female life history traits. In a meta-analysis covering 70 arthropod species, South and Lewis (2011) found that females receiving larger ejaculates benefit from increased fecundity at the expense of decreased life expectancy. In addition, females receiving ejaculates from multiple males experienced a greater reduction in lifespan than females that re-mated with the same male (South and Lewis, 2011). In the haplodiploid spider mite Tetranychus urticae, multiply inseminated females laid fewer fertilized eggs and produced fewer offspring compared to females that only mated once, suggesting that the fitness costs of polyandry extend beyond a mere reduction in lifespan (Rodrigues et al., 2020). Reviewing 20 years of cryptic female choice studies, Firman et al. (2017) proposed an evolutionary tight rope process, where females balance initial costs of discriminating between male ejaculates with later costs of reduced lifetime fertility. To date, research quantifying the potential trade-offs between female investments in fecundity and mate choice is still scarce (Alonzo and Pizzari, 2013; Perry et al., 2013). Furthermore, whether the physiological costs of polyandry generally result from female investments into maintaining viable sperm, or reflect female investments to resolve sexual conflicts resulting from aggressive, molecular interactions between ejaculates remains an unresolved question.

The queens of polyandrous social wasps, social bees and ants provide interesting model organisms to study sexual conflict vs. fecundity trade-offs, because selection for maintaining many viable spermatozoa is very strong (Boomsma et al., 2005), and polyandry evolved secondarily from monandrous ancestors, so that sexual conflicts evolved multiple times convergently (Hughes et al., 2008). Some social insect females store remarkably large numbers of viable sperm inside their spermathecae to last for years to decades (Pamilo, 1991; Keller and Genoud, 1997; Jemielity et al., 2005), as documented in the honey bee Apis mellifera (up to 5.5 million) (Baer et al., 2016), the fire ant Solenopisis invicta (up to 7 million) (Tschinkel and Porter, 1988) or the leaf-cutting ant Atta colombica (up to 465 million) (Baer et al., 2006). All social insect queens only mate during a short period early in their adult lives, during which they acquire their lifetime's supply of sperm (Wheeler and Krutzsch, 1994; Boomsma et al., 2005). Their cumulative fecundity is ultimately limited by the number of sperm they initially stored, rather than by the number of eggs they can produce (Kraus et al., 2004). Consequently, social insect queens have to be very economical about preserving stored sperm and using it prudently. Indeed, queens of honey bees, fire- and leaf-cutting ants are capable of reliably fertilizing eggs for many years, using only 1 or 2 sperm at a time (Tschinkel and Porter, 1988; den Boer et al., 2009; Baer et al., 2016).

Obligate polyandry evolved in multiple lineages of social Hymenoptera after permanent queen and worker castes arose (Boomsma and Ratnieks, 1996; Boomsma et al., 2005; Hughes et al., 2008; Boomsma, 2013). In many, but not all of these species, multiple mating is positively correlated with the number of sperm a female has stored (Fjerdingstad and Boomsma, 1998). In polyandrous leaf-cutting ants, male seminal fluid contains specific serine proteases, which are involved in reducing the viability of rival sperm (Dosselli et al., 2019). As can be expected, queens neutralize male-driven sexual conflict (Dosselli et al., 2019) immediately after mating, in order to minimize sperm loss, which would result in a reduction of their life time fecundity (Fjerdingstad and Boomsma, 1998).

Newly mated queens of Atta leaf-cutting ants initiate colonies on their own (claustrally) (Mintzer, 1990). With $40 \%$ body fat, Atta queens are the leanest claustral nest foundresses known in ants, and they produce their first generation of workers only on their fat reserves (Seal, 2009). Before they can even start to lay eggs though, they first have to excavate a nest burrow and initiate a fungus garden. To do this, queens have to upregulate their immune system to avoid contracting infections by surrounding soil microbes (Baer et al., 2006; Augustin et al., 2010), while at the same time storing and maintaining a maximal number of sperm. These energetic demands appear to trade off, because the ability of a newly mated A. colombica queen to upregulate her immune system is negatively correlated with the number of spermatozoa she has stored, and with the number of males she has mated with (Baer et al., 2006). The period of claustral colony initiation is linked to mortality rates of up to $95 \%$ (Weber, 1972; Fowler et al., 1986), as queens have to balance their immediate survival against their future fecundity (Baer and Boomsma, 2006). Ultimately though, how costly the removal of instantaneous hostility between ejaculates actually is relative to the longer-term challenge of securing survival and storage of maximal numbers of viable sperm remained ambiguous (Baer and Boomsma, 2006).

Here, we conducted an artificial insemination experiment that allowed us to separately test how sperm and seminal fluid affect several queen life history traits. In leaf-cutting ants ejaculates that damage female survival will have no pay-off for males, because contrary to solitary females, queens will not pass on male genes until several years after colony founding (Oster and Wilson, 2020). We therefore hypothesized that mating costs in leaf-cutting ants are primarily driven by physiological costs associated with storing and maintaining sperm, rather than by costs of neutralizing hostile interactions between competing ejaculates. To differentiate between these effects, we inseminated mature virgin queens of A. colombica with either full ejaculates, sperm only or seminal fluid only, and compared queen immune defenses relative to control treatments that neither exposed queens to sperm, nor to seminal fluid. We monitored queen survival and immunocompetence over the first crucial 6 days after insemination. Within this time frame, queens of leaf-cutting 
ants complete the physiological processes of sperm storage and neutralizing inter-ejaculate hostility (den Boer et al., 2010) while at the same time upregulating their immune system (Baer et al., 2006).

\section{MATERIALS AND METHODS}

\section{Collecting and Maintaining Female and Male Reproductives of Atta colombica}

We excavated sexually mature virgin queens and males of A. colombica from four different colonies in Gamboa, Republic of Panamá at the start of the rainy season in May 2012 and weighed each queen at the start (day 1) and at the end (day 6) of the experiment. This allowed us to obtain a queen's relative loss of weight by subtracting her end-weight from her start-weight and transforming this measure to a proportion by dividing absolute weight loss by initial body weight.

\section{Insemination Procedures}

We randomly allocated queens to one of five insemination treatments: (1) full ejaculates, (2) sperm only, (3) seminal fluid only, (4) Hayes saline solution or (5) a sham treatment. We anaesthetized each queen with $\mathrm{CO}_{2}$ for approximately $1 \mathrm{~min}$ before placing her into a modified queen holder connected to an insemination instrument normally used to inseminate honey bee queens, ${ }^{1}$ as described in earlier work (den Boer et al., 2013). We prepared samples of full ejaculates, sperm only or seminal fluid only for each queen by randomly picking sexually mature males from three colonies. We made sure that males used for sample collection were never related (i.e., from the same colony) to the queens used for any insemination treatment. To obtain ejaculates, we used a previously described method (den Boer et al., 2013), gently squeezing a male's abdomen and collecting approximately $1 \mu \mathrm{l}$ of ejaculate appearing at the end of the endophallus into a new glass capillary attached to a syringe. We then inseminated each queen with $10 \mu \mathrm{l}$ of mixed ejaculates originating from 10 males. To prepare seminal fluid, we pooled ejaculates from approximately 20 males collected as described above and centrifuged the sample twice at $13,500 \mathrm{rpm}$ for $10 \mathrm{~min}$ at room temperature, discarding the sperm pellets after each centrifugation step. We then used $10 \mu \mathrm{l}$ of seminal fluid to "inseminate" each queen. To inseminate queens with sperm only, we used a method described earlier (den Boer et al., 2013). In short, we anesthetized males with $\mathrm{CO}_{2}$, dissected out their accessory testes under a stereo-microscope and rinsed them twice in Hayes saline solution. Using a sterile injection needle, we pierced a small hole into the accessory testes and collected $1 \mu \mathrm{l}$ of outflowing sperm into a class capillary. We collected $1 \mu \mathrm{l}$ of sperm per male from a total of 10 males resulting in a total volume of $10 \mu \mathrm{l}$ of sperm used for inseminations. To control for possible effects of volume changes of the spermatheca, we "inseminated" an additional batch of queens with $10 \mu \mathrm{l}$ of Hayes saline solution each $(9.0 \mathrm{~g} / \mathrm{l} \mathrm{NaCl}, 0.2 \mathrm{~g} / \mathrm{l} \mathrm{CaCl}, 0.2 \mathrm{~g} / 1 \mathrm{KCl}, 0.1 \mathrm{~g} / \mathrm{l} \mathrm{NaHCO} 3)$

${ }^{1}$ http://www.besamungsgeraet.de/_en/
(Hayes, 1953). We finally used a second control treatment (shamtreatment) to test for possible effects of the artificial insemination procedure. To do this, we briefly placed a clean, empty glass capillary into the anesthetized queen's bursa copulatrix without injecting any sample.

\section{Monitoring Queen Survival}

After the insemination procedure, we placed each queen into an individual plastic petri-dish supplied with a ball of moist cotton, a small piece of fungus garden collected from her maternal colony (1-2 cm in diameter), and five small workers. We added these workers, because due to experimental reasons, the size of the fungus garden was larger compared to initial fungus gardens of queens at that stage (Pagnocca et al., 2008), and lone queens would not have been able to maintain that mass. However, this should not have impacted our data, because all queens were provided with the same amount of fungus garden and workers, and we were primarily interested in differences between treatments. We recorded queen survival each day, while always replenishing the piece of fungus garden and replacing dead workers as well as moist cotton balls. Petri-dishes were left in the dark at ambient conditions for 6 days.

\section{Measuring a Queen's Immune Response}

To assess the strength of the surviving queen's immune system, we measured her encapsulation response, a melanization reaction deployed against foreign objects inside an insect's haemolymph, such as the egg of a parasitoid. In a biochemical cascade, phenyl oxidase produces inole groups, which polymerize to melanize the foreign object. This process can be quantified (GonzálezSantoyo and Córdoba-Aguilar, 2012; Elizalde et al., 2019) and has been shown to be energetically costly and condition dependent (González-Santoyo and Córdoba-Aguilar, 2012). Here, we used a method developed in earlier work (Baer et al., 2006): We cut $1 \mathrm{~mm}$ pieces of fishing nylon $(0.13 \mathrm{~mm}$ diameter $)$ into a petri-dish filled with $70 \%$ ethanol. In a next step, we sedated queens with $\mathrm{CO}_{2}$ and placed them into an artificial insemination instrument, as described above. Using a thin, sterile injection needle, we pierced a small hole into the membrane between the 6th and 7th sternite and used INOX 5 watchmaker forceps to insert a piece of nylon into the haemocoel, after which we let the queens recover in their original petri dish. $24 \mathrm{~h}$ later, we freeze-killed the queens at-20 C and dissected out the implant under a Leica stereomicroscope. To measure the degree of melanization of each implant, we attached a digital camera (Canon D30) to a Leica stereomicroscope and took two pictures of each nylon implant at $62.5 \times$ magnification against a white background, and two images of their white background as a control. We transferred the images to a computer and transformed them to gray scale using the software ImageJ. ${ }^{2}$ We then calculated encapsulation response as the difference between the average gray value of the implant minus the average value of the control image of the background.

\footnotetext{
${ }^{2}$ https://imagej.nih.gov/ij/docs/menus/analyze.html: Mean Gray Value is the average gray value within the selected sampling quadrat, i.e., the sum of the gray values of all pixels registered divided by the number of pixels.
} 


\section{Measuring Spermatheca Size}

In order to estimate whether the queens were able to remove liquids or sperm cells from their spermathecae, we measured spermatheca size. To do this, we removed the spermathecae from each surviving queen in our experiment, using INOX 5 watchmaker forceps, rinsed them in Hayes saline and took two digital pictures with a Canon EOS 5 camera connected to a Leica stereomicroscope. Pictures were transferred to a computer and analyzed using ImageJ. We measured the diameter of each spermatheca in both photos and used the average of both measurements for statistical analyses.

\section{Statistical Analyses}

A total of 72 queens from four different colonies became available for statistical analyses, which included 16 inseminated with full ejaculates, 16 with sperm only and 14 with seminal fluid only. For the control treatments, we inseminated 14 queens with saline solution, and another 12 queens received the sham treatment. However, these original treatments could not be used directly for statistical tests to unravel the differential effects of sperm and seminal fluid on queen survival, because the full ejaculate treatment contained both seminal fluid and sperm. After an initial analysis of overall treatment effects, we therefore recoded the original data using two dummy variables, i.e., sperm presence or absence and seminal fluid presence or absence. The original treatments were then individually defined as full ejaculates $(1 / 1)$, sperm only (1/0), seminal fluid only (0/1) and controls $(0 / 0)$. For all further analyses, we then used these dummy variables "Contains Sperm" and "Contains Seminal Fluid" as factors and obtained separate $p$-values for the presence or absence of sperm or seminal fluid. Queens inseminated with full ejaculates or sperm were pooled into "Contains Sperm" $(N=32)$ while "inseminations" with seminal fluid only, Hayes saline controls or sham controls were pooled and categorized as "Without Sperm" $(N=40)$. For the "Contains Seminal Fluid" category, we grouped queens that had received full ejaculates or seminal fluid only $(N=30)$, and we grouped queens that had received either sperm only or one of the control treatments into "Without Seminal Fluid" $(N=42)$. Because lifespans and body weights, as well as encapsulation responses were statistically indistinguishable for queens that had received one of the control treatments, we pooled them into a single treatment group $(N=26)$.

We used IBM SPSS 27 for Macintosh for all our analyses. To test how queen survival was affected by individual insemination treatments and to compare their survival in the presence of sperm or seminal fluid, we calculated Kaplan-Meier survival probabilities using $p$-values from Breslow General Wilcoxon tests. For post-hoc analyses we used Cox Regressions. When indicated, we Bonferroni adjusted $p$-values, dividing the usual threshold $p$-value of 0.05 by 2 (the number of tests we had performed) to the new threshold $p$-value of 0.025 . To unravel the effects of the queens' initial body weight on their survival, depending on their exposure to sperm or seminal fluid, we used survival to 6 days $(0=$ no and $1=$ yes $)$ as a dependent variable in a GENLIN analysis for a binomial distribution. To analyze encapsulation responses in the presence of sperm or seminal fluid, and to compare spermatheca sizes between insemination treatments, we used GENLIN models with a gamma distribution and log-link.

\section{RESULTS}

In total, 47 (65\%) queens survived to the end of the experiment on day 6, but none of the queens had started to lay eggs at that point. We found a significant overall treatment effect on the survival probabilities of queens (Figure 1A), confirmed by Kaplan-Meier survival analysis $\left(N=72\right.$, Wald-Chi ${ }^{2}=16.024$, $\mathrm{df}=4, p=0.003)$. Comparing the queens' cumulative survival after insemination with sperm only $(N=16)$ with the pooled control treatments $(N=14$ for Hayes saline $+N=12$ for sham treatment $=N=26$ ) revealed that it was the presence of sperm in the queens' spermathecae that significantly shortened their lifespan (Figures 1A,B), as confirmed by post-hoc Cox Regression analysis $(N=42$, Wald $=8.127, \mathrm{df}=1, p=0.004)$. However, we did not find this effect for the presence of seminal fluid (Figure 1C), i.e., when comparing "inseminations" with seminal fluid only $(N=14)$ with the pooled control treatments $(N=14$ for Hayes saline +12 for sham treatment $=26)$ using a post-hoc Cox Regression $(N=40$, Wald $=0.155, \mathrm{df}=1$, $p=0.694$ ).

Using a GENLIN model with survival to 6 days (yes/no) as a dependent variable, we found a significant interaction between sperm presence and initial body weight (Table 1, GENLIN, $N=72, \mathrm{df}=1$, Wald-Chi Square $=4.809, p=0.028)$. For those queens that survived to the end of the experiment and had received sperm as part of their insemination treatment $(N=16$ for full ejaculates $+N=16$ for sperm only; total $N=32$ ), heavier queens were more likely to survive until the end of the experiment compared to lighter queens (Figure 2). We did not find such an effect when analyzing "insemination" treatments that did not involve sperm (i.e., seminal fluid $(N=14)$ or one of the Hayes saline $(N=14)$ or sham $(N=12)$ control treatments, i.e., total $N=26$ ), and the interaction term between seminal fluid "insemination" and a queen's body weight was not significant (Figure 2 and Table 1, GENLIN, $N=72$, $\mathrm{df}=1$, Wald-Chi Square $=0.992, p=0.319$ ).

Encapsulation responses were available for a subset of 23 surviving queens originating from three different colonies, after we excluded two queens with far too high outlier values for encapsulation response relative to expectation (Hoaglin and Iglewicz, 1987). We also excluded all queens from colony 1 , because none survived inseminations containing sperm followed by an encapsulation treatment until the last day of the experiment. The encapsulation responses of queens that had survived inseminations involving sperm $(N=10$, pooling $N=5$ for both full ejaculates and sperm only) decreased when queens had lost more weight during the experiment (Figure 3 and Table 2), as illustrated by the significant interaction term between sperm presence and relative weight loss in the Generalized Linear Model (GENLIN, $N=23$, Wald-Chi ${ }^{2}=4.191$, $\mathrm{df}=1, p=0.041$ ).

In survivors of "inseminations" that did not contain sperm (seminal fluid only $(N=4)$ or one of the control treatments 
( $N=5$ for Hayes saline and $N=4$ for sham controls-all pooled for analysis to $N=13$ ), we did not find a significant interaction effect between encapsulation response and weight loss (Figure 3 and Table 2, GENLIN, $N=23$, Wald $-\mathrm{Chi}^{2}=0.741$, $\mathrm{df}=1$, $p=0.389)$. However, encapsulation responses differed between colonies (Table 2, GENLIN, $N=23$, Wald-Chi ${ }^{2}=20.747, \mathrm{df}=2$, $p<0.0005)$, which might indicate that local environmental effects affected general offspring condition, or that parents were genetically different for immunocompetence responses under the experimental conditions that we offered.

Spermatheca sizes were available for a subset of 33 surviving queens from four colonies. Because we were unable to measure the empty spermathecae of $A$. colombica queens reliably, as they were folded up (Baer and Boomsma, 2006), we excluded them from the following analyses. After insemination with either full ejaculates $(N=7)$, sperm only $(N=5)$, seminal fluid only $(N=10)$ or Hayes saline $(N=11)$, the spermathecae of A. colombica queens always unfolded because they were filled, but the extent to which this happened varied widely between treatments (Figure 4A and Table 3A, Table 3B), as confirmed by a Generalized Linear Model (GENLIN, $N=33$, Wald$\left.\mathrm{Chi}^{2}=33.970, \mathrm{df}=3, p<0.0005\right)$. Given that spermathecal size is a morphological trait, rather than a physiological condition trait such as encapsulation response, we expected no differences across the four colonies from which queens were sampled, which was confirmed in formal analysis (Table 3A, Table 3B, GENLIN, $N=33$, Wald-Chi ${ }^{2}=1.973, \mathrm{df}=3, p=0.578$ ).

The impression that the two left bars in Figure $4 \mathrm{~A}$ were much higher that the two right bars was confirmed in a pooled dummy variable analysis. This revealed that inseminations containing sperm $(N=7$ for full ejaculates and $N=5$ for sperm only, giving a total of $N=12$ ) disproportionally increased spermatheca sizes in surviving queens (Figure 4B and Table 3A, Table 3B, GENLIN, $N=33$, Wald-Chi ${ }^{2}=21.614, \mathrm{df}=1, p<0.0005$ ), relative to "inseminations" containing seminal fluid or control liquids (total $N=21$, Wald-Chi ${ }^{2}=5.103, \mathrm{df}=1, p<0.024$, Bonferronicorrected threshold $p=0.025)$. The latter result indicates, that seminal fluid is slightly more likely to be stored than Hayes saline, while the former result confirms that true inseminations involving sperm fill the spermatheca much more consistently. Again, we did not find an effect of the colonies queens had been sampled from (Wald-Chi ${ }^{2}=2.654, \mathrm{df}=3, p=0.448$ ).

\section{DISCUSSION}

We conducted an artificial insemination experiment to test, how the presence of sperm or seminal fluid in the sexual tract of freshly mated queens of $A$. colombica affects their survival in the first week of independent life, which corresponds to the most critical
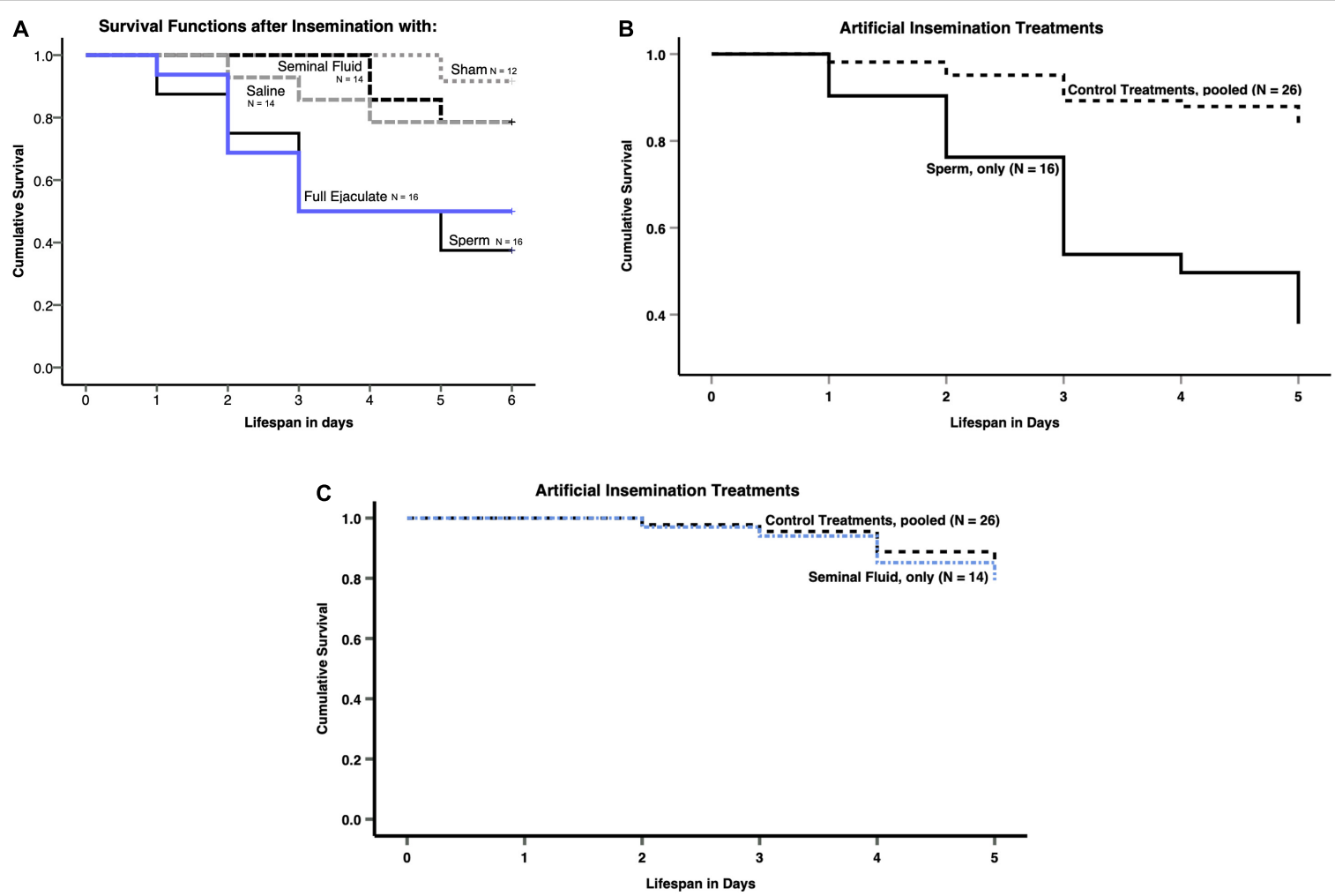

FIGURE 1 | (A) Kaplan-Meier survival functions of Atta colombica queens after artificial insemination with either: full ejaculates, sperm only, seminal fluid only, a Hayes saline solution, or a sham treatment. (B) Comparison of Kaplan-Meier survival functions of Atta colombica queens after pooling treatments, so they could be subdivided in inseminations involving sperm and "inseminations" not involving sperm. (C) Comparison of Kaplan-Meier survival functions of Atta colombica queens after pooling treatments, so they could be subdivided in "inseminations" involving seminal fluid and inseminations not involving seminal fluid. 
TABLE 1 | Comparing inseminations involving sperm ( $N=16$ for full ejaculates and $N=16$ for sperm only, giving a total of $N=32$ ) and "inseminations" involving no sperm ( $N=14$ for both seminal fluid only and Hayes saline, and $N=12$ for sham treatment, giving a total of $N=40$ ), produced a significant main effect of sperm presence and a significant interaction term between sperm presence and body weight, indicating that heavier queens of $A$. colombica were more likely to survive to the end of the experiment than lighter ones. After "insemination" involving seminal fluid, we neither found a significant main effect nor a significant interaction term with body weight (Figure 2).

\begin{tabular}{|c|c|c|c|}
\hline \multirow{2}{*}{$\begin{array}{l}\text { GENLIN: Dependent Variable } \\
\text { Survival to } 6 \text { days yes/no, } \\
N=72 \text { queens from } 4 \text { colonies }\end{array}$} & \multicolumn{3}{|c|}{ Type III } \\
\hline & Wald-Chi ${ }^{2}$ & df & Sig. \\
\hline (Intercept) & 2.088 & 1 & 0.148 \\
\hline Sperm presence & 7.374 & 1 & 0.007 \\
\hline Seminal fluid presence & 0.762 & 1 & 0.383 \\
\hline Colony & 5.331 & 3 & 0.149 \\
\hline Body weight & 3.307 & 1 & 0.069 \\
\hline Sperm presence * body weight & 4.809 & 1 & 0.028 \\
\hline Seminal fluid presence * body weight & 0.992 & 1 & 0.319 \\
\hline Goodness of fit ${ }^{a}$ & Value & df & \\
\hline Scaled deviance & 63.230 & 62 & \\
\hline
\end{tabular}

alnformation criterion for which smaller values indicate a better fit. Bold values highlight significant p-values.

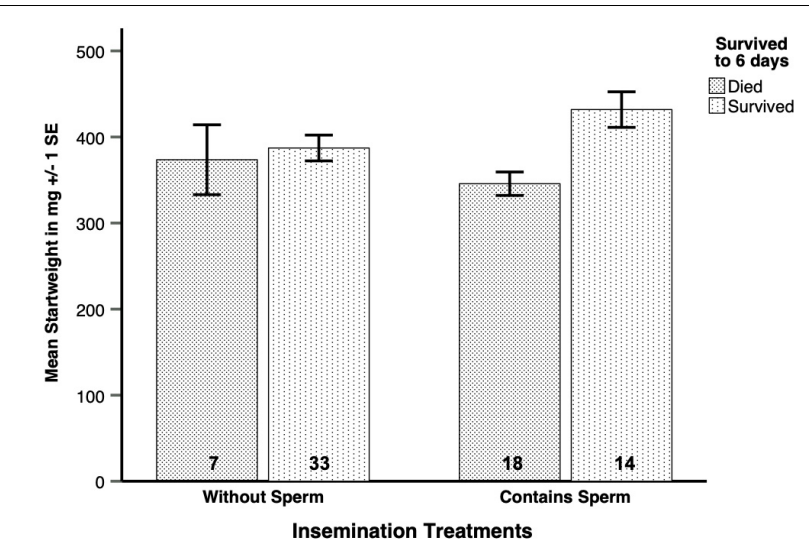

FIGURE 2 | After insemination with full ejaculates or sperm only, heavier queens of Atta colombica were more likely to survive until the end of the experiment than lighter ones. This interaction between body weight and survival was not significant for queens that had received a treatment not involving sperm (Table 1)

time in the life history of an A. colombica queen. In Atta species, the trade-off between eliminating aggressive, sexually selected male traits (to prevail in sperm competition) and naturally selected traits to preserve optimal numbers of high-quality sperm (to the benefit of a queen and all her mates), is expected to be particularly acute during this first week of solitary nest-founding directly following insemination (Baer et al., 2006). We empirically tested whether previously documented insemination costs for queens primarily originate from physiological expenditures associated with the storage and maintenance of sperm, or from female-controlled physiological processes to deactivate malemale hostility components in seminal fluid.

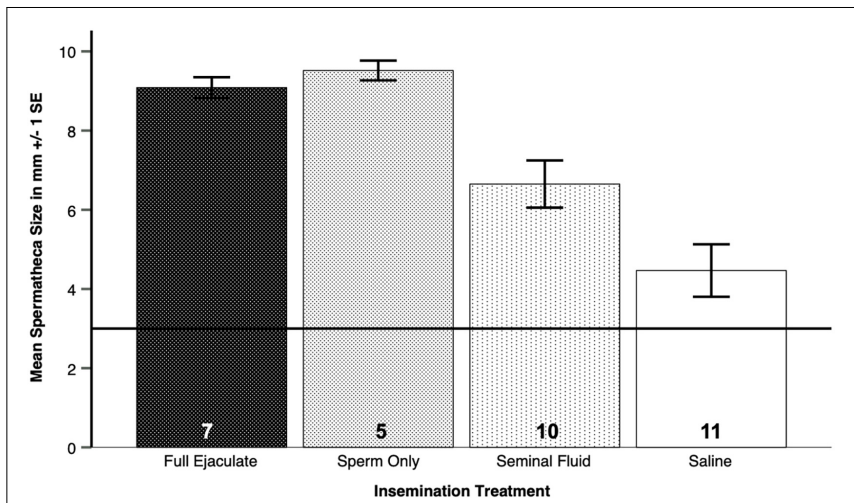

FIGURE 3 | The spermatheca sizes of surviving Atta colombica queens inseminated with either full ejaculates, sperm only, seminal fluid only or a Hayes saline solution varied widely and significantly (Table 3A). The horizontal line is the approximate diameter of empty spermathecae in A. colombica queens.

TABLE 2 | For queens of Atta colombica that had survived inseminations involving sperm ( $N=5$ for both full ejaculates and sperm only ejaculates, pooled here for analysis to $N=10$ ), encapsulation responses decreased, the more weight queens had lost during the experiment, as indicated by the significant interaction term between sperm presence and relative weight loss (Figure $\mathbf{3}$ ) in addition to a significant main effect of sperm presence. Survivors of inseminations with seminal fluid only ( $N=4$ ) or one of the control treatments ( $N=5$ for Hayes saline or $N=4$ for sham controls - all pooled here to $N=13$ ), showed no significant interaction with weight loss (Figure 3). The encapsulation response also differed significantly across the three colonies from which queens were sampled.

\begin{tabular}{|c|c|c|c|}
\hline \multirow{2}{*}{$\begin{array}{l}\text { GENLIN: Dependent Variable } \\
\text { Encapsulation Response, } \\
N=23 \text { queens from } 3 \text { colonies }\end{array}$} & \multicolumn{3}{|c|}{ Type III } \\
\hline & Wald-Chi ${ }^{2}$ & df & Sig. \\
\hline (Intercept) & 229.050 & 1 & $p<0.0005$ \\
\hline Sperm presence & 5.601 & 1 & 0.018 \\
\hline Seminal fluid presence & 0.100 & 1 & 0.751 \\
\hline Colony & 20.747 & 2 & $p<0.0005$ \\
\hline Relative weight loss & 7.753 & 1 & 0.005 \\
\hline $\begin{array}{l}\text { Sperm presence * relative weight } \\
\text { loss }\end{array}$ & 4.191 & 1 & 0.041 \\
\hline $\begin{array}{l}\text { Seminal fluid presence * relative weight } \\
\text { loss }\end{array}$ & 0.741 & 1 & 0.389 \\
\hline Goodness of fit ${ }^{a}$ & Value & df & \\
\hline Scaled deviance & 23.853 & 15 & \\
\hline
\end{tabular}

a Information criterion for which smaller values indicate a better fit. Bold values highlight significant $p$-values.

We found that sperm-storing queens were less likely to survive during the 6 days following insemination than queens that had received sperm-less "inseminations" with only seminalfluid or a control treatment. Moreover, surviving spermexposed queens were heavier than inseminated queens that died during the experiment, and these higher weights were associated with higher encapsulation responses, consistent with this immune response being costly. In earlier work on the ant Lasius niger Chérasse and Aron showed that the viability of stored sperm is-as expected-not affected by an immune 

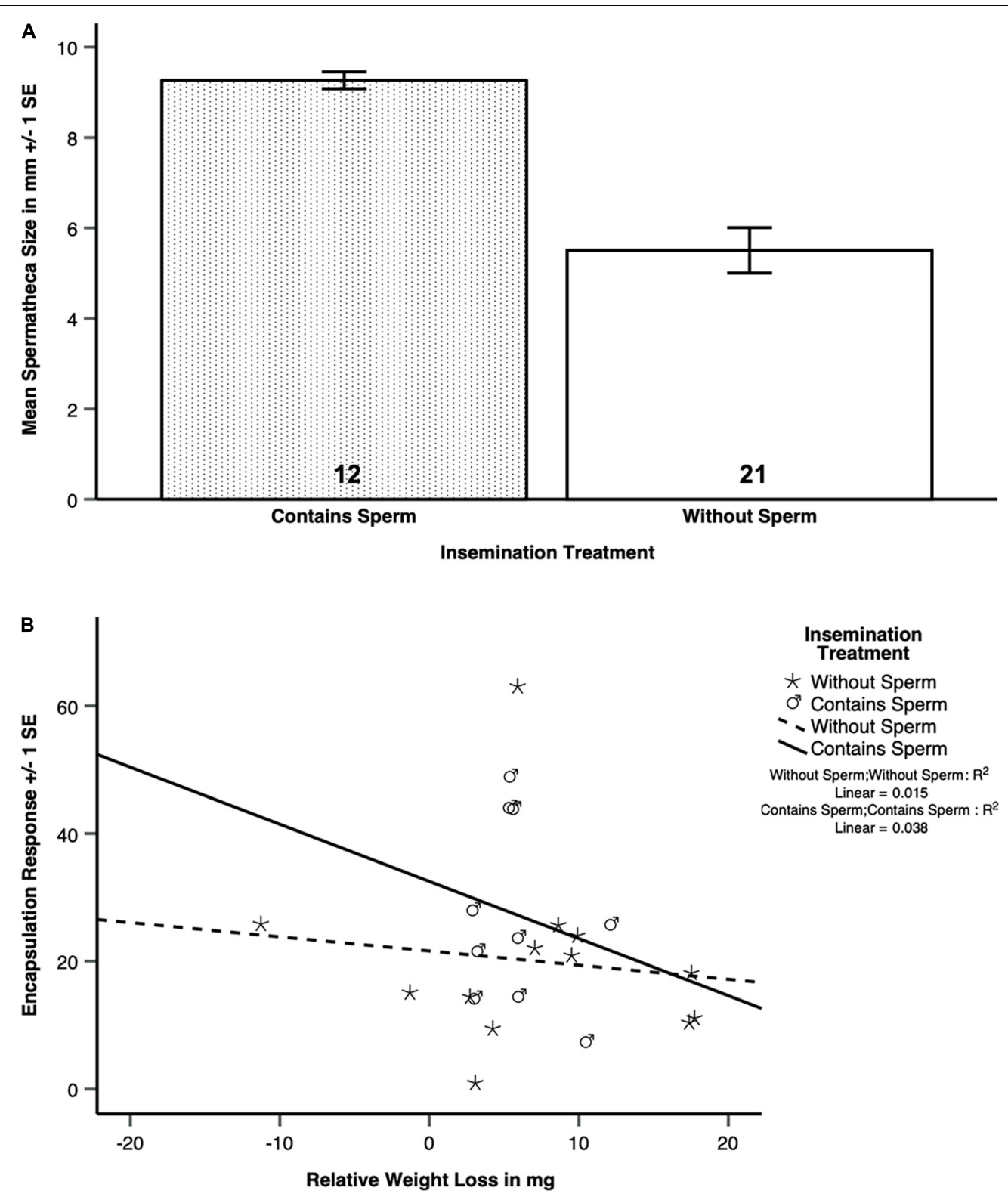

FIGURE 4 | (A) The spermathecal sizes of surviving Atta colombica queens after inseminations with full ejaculates or sperm only (pooled effects) were significantly larger than those of queens inseminated with Hayes saline solution or seminal fluid only (Table $\mathbf{3 B}$ ). (B) Among the queens of Atta colombica that had survived an insemination treatment that involved sperm, those that had lost more weight had a significantly lower encapsulation response than those that had lost less weight. This interaction was not significant for survivors of treatments not involving sperm (Table 2).

challenge of queens, because queens prioritize sperm viability over other life history traits. Furthermore, Lasius queens which expressed stronger immune activation also lost more body weight, supporting our finding that immune responses are costly (Chérasse and Aron, 2018). Further support for this conclusion comes from González-Santoyo and Córdoba-Aguilar (2012) who showed, that mounting an encapsulation response is conditiondependent, as expected when incurring high energetic costs. However, for the Atta queens that we had inseminated with seminal fluid only, we neither found an effect of body weight on survival, nor an effect of weight-loss on encapsulation response.
Together, these observations indicate that-contrary to findings in promiscuous solitary insects (Chapman et al., 1993, 1995; Snook and Hosken, 2004) - the highest post-mating costs for queens of $A$. colombica are incurred right after copulation, because queens have to initiate their colonies solitarily and will not have workers to provision them for 2-3 months. The immediate costs after insemination emanate from the need to initiate physiological changes (e.g., ovary development) to prepare for laying eggs and keeping large amounts of sperm alive in the spermatheca (Baer et al., 2006; Augustin et al., 2010), but not or hardly from neutralizing sperm 
competition inside the spermatheca (Dosselli et al., 2019). In honey bees, the cost of sperm storage is linked to significant changes in the spermathecal fluid proteome (Baer et al., 2009), suggesting that this process incurs considerable costs across the social insects.

Based on the special biology of ants, our findings are not surprising. Leaf-cutting ant queens achieve remarkable levels of fecundity: A. colombica queens store up to 465 million sperm after a single nuptial flight (Fjerdingstad and Boomsma, 1998; Baer et al., 2006), which allows some of their colonies to grow to several million workers (Weber, 1972; Villesen et al., 1999, 2002). Mated queens keep their stored sperm viable (den Boer et al., 2008) during a reproductive lifespan of up to 28 years (Weber, 1972; Keller and Genoud, 1997; Fjerdingstad and Boomsma, 1998), and they continue to produce fertilized eggs by using very few sperm per egg even though senescence makes older queens somewhat less precise (den Boer et al., 2009). Even so, the reproductive success of $A$. colombica queens is likely to be ultimately sperm-limited, and their colonies require several years of initial, purely somatic (worker) growth before being able to produce males and the first virgin queens (gynes) (Oster and Wilson, 2020). It seems therefore logical that molecular mechanisms of antagonism between the seminal fluids of rival ejaculates (Dosselli et al., 2019) have not been selected to become so aggressive as to compromise a queen's health before she can pass on any genes to future generations (Baer and Boomsma, 2004). If termination of sperm competition is compulsory, queens might use this mechanism to optimize the number of live sperm stored in trade-off with other life history traits, such as immunity. It would also serve the interests of all parties that these reproductive decisions are implemented quickly and at minimal costs. Whether the spermathecal fluids known to be enriched with protease inhibitors that queens actively mix with newly stored sperm and seminal fluid (Dosselli et al., 2019) are maximally cost-effective is unknown. However, high efficiency in this specific function is consistent with the selection scenario outlined above, and should also be expected to have evolved, albeit not necessarily via the same control mechanisms, in other polyandrous social insect queens.

In contrast to the likely minor cost of sexual conflict, maintaining stored sperm will always incur high costs, because queens are under selection to store as much sperm as they can obtain during their brief mating window, until the survival costs during the lone colony founding stage become prohibitive (Baer et al., 2006). We can therefore not expect that this cost component will ever be low. In the honey bee Apis mellifera for example, a metabolic network of more than 120 proteins provides stored sperm cells with energy, reduces their oxidative stress, and protects them from microbial attack (Baer et al., 2009).

In many ant species, queens are facultatively polyandrous or use sperm of a single male to sire all their lifetime offspring (Boomsma and Ratnieks, 1996; Boomsma et al., 2009; Boomsma, 2013). However, Atta queens always mate with multiple males (Villesen et al., 1999, 2002) and receive ejaculates directly into the spermatheca during insemination (Baer and Boomsma, 2006; den Boer et al., 2015). This is unusual, because other social hymenopteran queens, including those
TABLE 3A | Spermathecal sizes (diameters) of Atta colombica queens that had survived one of the insemination treatment, i.e., full ejaculates $(N=7)$, sperm only $(N=5)$, seminal fluid only $(N=10)$ or Hayes saline solution $(N=11)$ varied widely between treatments (Figure 4A), but we found no difference in spermatheca sizes across the four colonies from which queens were sampled.

\begin{tabular}{|c|c|c|c|}
\hline \multirow{2}{*}{$\begin{array}{l}\text { GENLIN: Dependent variable } \\
\text { Spermatheca size, } \\
N=33 \text { queens from } 4 \text { colonies }\end{array}$} & \multicolumn{3}{|c|}{ Type III } \\
\hline & Wald - Chi ${ }^{2}$ & df & Sig. ${ }^{a}$ \\
\hline (Intercept) & 1274.415 & 1 & $<0.0005$ \\
\hline Insemination treatment & 33.970 & 3 & $<0.0005$ \\
\hline Colony & 1.973 & 3 & 0.578 \\
\hline Goodness of fit ${ }^{b}$ & Value & df & \\
\hline Scaled deviance & 33.459 & 26 & \\
\hline
\end{tabular}

For this analysis, we did not evaluate the sham-treated queens.

${ }^{a}$ Bonferroni-corrected threshold of significance $p=0.025$.

bInformation criterion for which smaller values indicate a better fit. Bold values highlight significant p-values.

TABLE 3B | Spermathecal sizes (diameters) of Atta colombica queens that had survived an insemination treatment involving sperm $(N=7$ for full ejaculates and $N=5$ for sperm only - here pooled for analysis to $N=12$ ) were significantly bigger than those of queens inseminated with seminal fluid $(N=10)$ or Hayes saline $(N=11)$, here pooled for analysis to $N=21$ (Figure 4B).

\begin{tabular}{|c|c|c|c|}
\hline \multirow{2}{*}{$\begin{array}{l}\text { GENLIN: Dependent variable } \\
\text { Spermatheca size, } \\
N=33 \text { queens from } 4 \text { colonies }\end{array}$} & \multicolumn{3}{|c|}{ Type III } \\
\hline & Wald-Chi' ${ }^{2}$ & df & Sig. ${ }^{a}$ \\
\hline (Intercept) & 1162.910 & 1 & $<0.0005$ \\
\hline Sperm presence & 21.614 & 1 & $<0.0005$ \\
\hline Seminal fluid presence & 5.103 & 1 & 0.024 \\
\hline Colony & 2.654 & 3 & 0.448 \\
\hline Goodness of fit ${ }^{b}$ & Value & df & \\
\hline Scaled deviance & 33.506 & 27 & \\
\hline
\end{tabular}

After insemination treatments involving seminal fluid, queen spermathecae were bigger than after inseminations with saline solution (Figure 4A). We found no difference in spermatheca sizes across the four colonies from which queens were sampled. We did not evaluate the sham-treated queens in this analysis. ${ }^{a}$ Bonferroni-corrected significance level $p=0.025$.

${ }^{b}$ Information criterion for which smaller values indicate a better fit. Bold values highlight significant p-values.

of the sister genus Acromyrmex (Liberti et al., 2018) receive sperm into the bursa copulatrix or the lateral oviducts first, before it is stored during a secondary process. Such two-step sperm storage procedures offer more opportunities for postcopulatory sexual selection, and may allow more sperm to be competitively removed without damaging queen health. In honey bees for example, only $3-5 \%$ of the 6 million sperm received during copulations will eventually become stored in a queen's spermatheca (Baer, 2005).

Although our findings are consistent with our initial expectation, further work is needed to evaluate, whether some of the responses documented here may be condition-dependent or non-additive. For example, it might be that spermathecal gene expression has co-evolved with the presence of both sperm and seminal fluid, so that isolating these two effects in separate treatments may not quite emulate the naturally 
occurring physiological responses. A follow-on experiment in which sperm and seminal fluid are inseminated in different but non-zero quantities would likely allow this issue to be resolved, but was beyond the scope of the present experiments.

\section{DATA AVAILABILITY STATEMENT}

The raw data supporting the conclusions of this article will be made available by the authors, without undue reservation.

\section{AUTHOR CONTRIBUTIONS}

BB-I: data evaluation and writing. SDB: experimental design, field work, and funding. JJB: revision, senior advisor, and funding. BB: supervision, experimental design, writing, and funding. All authors contributed to the article and approved the submitted version.

\section{REFERENCES}

Alonzo, S. H., and Pizzari, T. (2013). Selection on female remating interval is influenced by male sperm competition strategies and ejaculate characteristics. Philos. Trans. R. Soc. B 368:20120044. doi: 10.1098/rstb.2012.0044

Augustin, J. O., Santos, J. F., and Elliot, S. (2010). A behavioral repertoire of Atta sexdens (Hymenoptera, Formicidae) queens during the claustral founding and ergonomic stages. Insect. Soc. 58, 197-206.

Avila, F. W., Sirot, L. K., LaFlamme, B. A., Rubinstein, C. D., and Wolfner, M. F. (2011). Insect seminal fluid proteins: identification and function. Annu. Rev. Entomol. 56, 21-40. doi: 10.1146/annurev-ento-120709-144823

Baer, B. (2005). Sexual selection in Apis bees. Apidologie 36, 187-200.

Baer, B., and Boomsma, J. J. (2004). Male reproductive investment and queen mating frequency in fungus growing ants. Behav. Ecol. 15, 426-432.

Baer, B., and Boomsma, J. J. (2006). Mating biology of the leaf-cutting ants Atta colombica and A. cephalotes. J. Morphol. 267, 1165-1171. doi: 10.1002/jmor. 10467

Baer, B., Armitage, S. A. O., and Boomsma, J. J. (2006). Sperm storage induces an immunity cost in ants. Nature 441, 872-875. doi: 10.1038/nature04698

Baer, B., Collins, J., Maalaps, K., and den Boer, S. P. (2016). Sperm use economy of honeybee (Apis mellifera) queens. Ecol. Evol. 6, 2877-2885. doi: 10.1002/ece3. 2075

Baer, B., Eubel, H., Taylor, N. L., O’Toole, N., and Millar, A. H. (2009). Insights into female sperm storage from the spermathecal fluid proteome of the honeybee Apis mellifera. Genome Biol. 10:R67. doi: 10.1186/gb-2009-10-6-r67

Birkhead, T. R., and Moller, A. P. (1998). Sperm Competition And Sexual Selection. New York, NY: Academic Press.

Boomsma, J. J. (2013). Beyond promiscuity: mate-choice commitments in social breeding. Philosophical Trans. R. Soc. B Biol. Sci. 368:20120050. doi: 10.1098/ rstb.2012.0050

Boomsma, J. J., and Ratnieks, F. L. W. (1996). Paternity in eusocial Hymenoptera. Philos. Trans. R. Soc. B 351, 947-975.

Boomsma, J. J., Baer, B., and Heinze, J. (2005). The evolution of male traits in social insects. Annu. Rev. Entomol. 50, 395-420.

Boomsma, J. J., Kronauer, D. J. C., and Pedersen, J. S. (2009). “The evolution of social insect mating systems," in Organization Of Insect Societies - From Genome To Sociocomplexity, eds J. Gadau and J. Fewell (Cambridge, MA: Harvard University Press), 3-25.

Chapman, T., Hutchings, J., and Partridge, L. (1993). No reduction in the cost of mating for Drosophila melanogaster females mating with spermless males. Proc. R. Soc. Lond. B Biol. Sci. 253, 211-217. doi: 10.1098/rspb.1993. 0105

\section{FUNDING}

This study was funded by a Queen Elizabeth II Fellowship, a Future Fellowship, and a Discovery Project from the Australian Research Council (ARC) to BB, a start-up-grant to $\mathrm{BB}$ offered by the University of California Riverside, a Centre of Excellence grant by the Danish National Research Foundation (DNRF57) and an Advanced ERC grant to JJB, and the Marie-Curie International Outgoing Fellowship to SDB.

\section{ACKNOWLEDGMENTS}

We thank the Smithsonian Tropical Research Institute in Panama for facilities and logistical support to work in Gamboa, and the Autoridad Nacional de Ambiente (ANAM) in Panama for issuing collection and export permits for ant samples to Denmark and Australia.

Chapman, T., Liddle, L. F., Kalb, J. M., Wolfner, M. F., and Partridge, L. (1995). Cost of mating in Drosophila melanogaster females is mediated by male accessory gland products. Nature 373, 241-244. doi: 10.1038/373241a0

Chérasse, S., and Aron, S. (2018). Impact of immune activation on stored sperm viability in ant queens. Proc. Biol. Sci. 285:20182248. doi: 10.1098/rspb.2018. 2248

den Boer, S. P. A., Baer, B., and Boomsma, J. J. (2010). Seminal fluid mediates ejaculate competition in social insects. Science 327, 1506-1509. doi: 10.1126/ science.1184709

den Boer, S. P. A., Baer, B., Dreier, S., Aron, S., Nash, D. R., and Boomsma, J. J. (2009). Prudent sperm use by leafcutter ant queens. Proc. R. Soc. Lond. B Biol. Sci. 276, 3945-3953. doi: 10.1098/rspb.2009.1184

den Boer, S. P. A., Boomsma, J. J., and Baer, B. (2008). Seminal fluid enhances sperm viability in the leafcutter ant Atta colombica. Behav. Ecol. Sociobiol. 62, 1843-1849.

den Boer, S. P. A., Boomsma, J. J., and Baer, B. (2013). A technique to artificially inseminate leafcutter ants. Insect. Soc. 60, 111-118.

den Boer, S. P. A., Stürup, M., Boomsma, J. J., and Baer, B. (2015). The ejaculatory biology of leafcutter ants. J. Insect Physiol. 74, 56-62. doi: 10.1016/j.jinsphys. 2015.02.006

Dosselli, R., Grassl, J., den Boer, S. P. A., Kratz, M., Moran, J. M., Boomsma, J. J., et al. (2019). Protein-level interactions as mediators of sexual conflict in ants. Mol. Cell. Proteomics 18, S34-S45. doi: 10.1074/mcp.RA118.000941

Eberhard, W. G. (1996). Female Control: Sexual Selection By Cryptic Female Choice. Princeton, NJ: Princeton University Press.

Elizalde, L., Treanor, D., Pamminger, T., and Hughes, W. O. H. (2019). Immunity of leaf-cutting ants and its role in host-parasitoid relationships. J. Insect Physiol. 116, 49-56. doi: 10.1016/j.jinsphys.2019.04.008

Firman, R. C., Gasparini, C., Manier, M. K., and Pizzari, T. (2017). Postmating female control: 20 years of cryptic female choice. Trends Ecol. Evol. 32, 368-382. doi: 10.1016/j.tree.2017.02.010

Fjerdingstad, E. J., and Boomsma, J. J. (1998). Multiple mating increases the sperm stores of Atta colombica leafcutter ant queens. Behav. Ecol. Sociobiol. 42, 257-261.

Fowler, H. G., Pereira da Silva, V., Forti, L. C., and Saes, N. B. (1986). "Population dynamics of leaf-cutting ants: a brief review," in Fire Ants And LeafCutting Ants: Biology And Management, eds C. S. Lofgren and R. K. Vander Meer (Boulder, CO: Westview Press), 123-145.

González-Santoyo, I., and Córdoba-Aguilar, A. (2012). Phenoloxidase: a key component of the insect immune system. Entomol. Exp. Appl. 142, 1-16.

Hayes, E. O. (1953). Determination of a physiological saline solution for Aedes aegypti (L.). J. Econ. Entomol. 46, 624-627. 
Hoaglin, D. C., and Iglewicz, B. (1987). Fine-tuning some resistant rules for outlier labeling. J. Am. Stat. Assoc. 82, 1147-1149.

Hughes, W. O. H., Oldroyd, B. P., Beekman, M., and Ratnieks, F. L. W. (2008). Ancestral monogamy shows kin selection is key to the evolution of eusociality. Science 320, 1213-1216. doi: 10.1126/science.1156108

Jemielity, S., Chapuisat, M., Parker, J. D., and Keller, L. (2005). Long live the queen: studying aging in social insects. Age (Dordr) 27, 241-248. doi: 10.1007/s11357005-2916-z

Keller, L., and Genoud, M. (1997). Extraordinary lifespans in ants: a test of evolutionary theories of ageing. Nature 389, 958-960.

Kraus, F. B., Neumann, P., van Praagh, J., and Moritz, R. F. (2004). Sperm limitation and the evolution of extreme polyandry in honeybees (Apis mellifera L.). Behav. Ecol. Sociobiol. 55, 494-501.

Kubli, E. (2003). Sex-peptides: seminal peptides of the Drosophila male. CMLS Cell. Mol. Life Sci. 60, 1689-1704. doi: 10.1007/s00018-003-3052

Liberti, J., Baer, B., and Boomsma, J. J. (2018). Rival seminal fluid induces enhanced sperm motility in a polyandrous ant. BMC Evol. Biol. 18:28. doi: 10.1186/ s12862-018-1144-y

Løvlie, H., Gillingham, M. A. F., Worley, K., Pizzari, T., and Richardson, D. S. (2013). Cryptic female choice favours sperm from major histocompatibility complex-dissimilar males. Proc. R. Soc. B 280:20131296. doi: 10.1098/rspb.2013. 1296

Mintzer, A. C. (1990). Colony foundation in leaf cutting ants: the perils of polygyny in Atta laevigata (Hymenoptera: Formicidae). Psyche (Stuttg.) 98, 1-5.

Oster, G. F., and Wilson, E. O. (2020). Caste And Ecology In The Social Insects. (MPB-12), Vol. 12. Princeton, NJ: Princeton University Press.

Pagnocca, F. C., Rodrigues, A., Nagamoto, N. S., and Bacci, M. (2008). Yeasts and filamentous fungi carried by the gynes of leaf-cutting ants. Antonie Van Leeuwenhoek 94, 517-526. doi: 10.1007/s10482-008-9268-5

Pamilo, P. (1991). Life span of queens in the ant Formica exsecta. Insect. Soc. 38, 111-119.

Parker, G. A. (1970). Sperm competition and its evolutionary consequences in the insects. Biol. Rev. 45, 525-567. doi: 10.3390/cells10020287

Parker, G. A., and Pizzari, T. (2010). Sperm competition and ejaculate economics. Biol. Rev. 85, 897-934. doi: 10.1111/j.1469-185X.2010.00140.x

Perry, J. C., Sirot, L., and Wigby, S. (2013). The seminal symphony: how to compose an ejaculate. Trends Ecol. Evol. 28, 414-422. doi: 10.1016/j.tree.2013.03.005

Pizzari, T., and Birkhead, T. R. (2000). Female feral fowl eject sperm of subdominant males. Nature 405, 787-789. doi: 10.1038/35015558

Poiani, A. (2006). Complexity of seminal fluid: a review. Behav. Ecol. Sociobiol. 60, 289-310.

Rodrigues, L. R., Figueiredo, A. R. T., Van Leeuwen, T., Olivieri, I., and Magalhães, S. (2020). Costs and benefits of multiple mating in a species with first-male sperm precedence. J. Anim. Ecol. 89, 1045-1054. doi: 10.1111/1365-2656.13171
Seal, J. N. (2009). Scaling of body weight and fat content in fungus-gardening ant queens: does this explain why leaf-cutting ants found claustrally? Insect. Soc. 56, $135-141$.

Simmons, L., and Siva-Jothy, M. T. (1998). "Sperm competition in insects: mechanisms and the potential for selection," in Sperm Competition and Sexual Selection, eds T. R. Birkhead and A. Moeller (London: Academic Press), 341434.

Snook, R. R., and Hosken, D. J. (2004). Sperm death and dumping in Drosophila. Nature 428, 939-941. doi: 10.1038/nature02455

South, A., and Lewis, S. M. (2011). The influence of male ejaculate quantity on female fitness: a meta-analysis. Biol. Rev. Camb. Philos. Soc. 86, 299-309. doi: 10.1111/j.1469-185X.2010.00145.x

Tschinkel, W. R., and Porter, S. D. (1988). Efficiency of sperm use in queens of the fire ant, Solenopsis invicta (Hymenoptera: Formicidae). Ann. Entomol. Soc. Am. $81,777-781$.

Villesen, P., Gertsch, P. J., Frydenberg, J., Mueller, U. G., and Boomsma, J. J. (1999). Evolutionary transition from single to multiple mating in fungus-growing ants. Mol. Ecol. 8, 1819-1825. doi: 10.1046/j.1365-294x.1999.00767.x

Villesen, P., Murakami, T., Schultz, T. R., and Boomsma, J. J. (2002). Identifying the transition between single and multiple mating of queens in fungus-growing ants. Proc. R. Soc. B 269, 1541-1548. doi: 10.1098/rspb.2002.2044

Weber, N. A. (1972). Gardening Ants: The Attines. Philadelphia, PA: The American Philosophical Society.

Wheeler, D. E., and Krutzsch, P. H. (1994). Ultrastructure of the spermatheca and its associated gland in the ant Crematogaster opuntiae (Hymenoptera, Formicidae). Zoomorphology 114, 203-212.

Conflict of Interest: The authors declare that the research was conducted in the absence of any commercial or financial relationships that could be construed as a potential conflict of interest.

Publisher's Note: All claims expressed in this article are solely those of the authors and do not necessarily represent those of their affiliated organizations, or those of the publisher, the editors and the reviewers. Any product that may be evaluated in this article, or claim that may be made by its manufacturer, is not guaranteed or endorsed by the publisher.

Copyright (C) 2022 Baer-Imhoof, den Boer, Boomsma and Baer. This is an openaccess article distributed under the terms of the Creative Commons Attribution License (CC BY). The use, distribution or reproduction in other forums is permitted, provided the original author(s) and the copyright owner(s) are credited and that the original publication in this journal is cited, in accordance with accepted academic practice. No use, distribution or reproduction is permitted which does not comply with these terms. 